\title{
Incidencia de la profundidad de muestreo sobre el contenido de formas de carbono y nitrógeno en suelos de la Zona Bananera del departamento del Magdalena (Colombia) ${ }^{1}$
}

\section{Incidence of the sampling depth on the content of forms of carbon and nitrogen in soils of the Zona Bananera of the department of Magdalena (Colombia)}

\author{
D. V. Escorcia, S. E. Aguirre y J. J. Vargas
}

Recibido: marzo 2 de 2021- Aceptado: diciembre 31 de 2021

\begin{abstract}
Resumen- El objetivo del trabajo fue determinar la incidencia de la profundidad de muestreo sobre el contenido de carbono y nitrógeno en suelos del municipio de Zona Bananera del departamento del Magdalena (Colombia). El análisis de los datos se realizó con software estadístico Infostat v.2008 y $R$ v.3.5.2, empleando análisis de varianza multivariado, análisis de correlación de Pearson y estadística descriptiva de cada variable. Los resultados muestran diferencias significativas $(p<0,05)$ entre los grupos de profundidad para las variables seleccionadas encontrándose contenidos menores en la profundidad 2. Además, existe una contribución significativa de las variables a las diferencias estadísticas entre los grupos, exceptuando el amonio y el nitrato. Se evidencia correlación entre las variables $\mathrm{C}$ oxidable/MO, $\mathrm{NO}_{3}{ }^{-} / \mathrm{NO}_{2}{ }^{-}, \mathrm{y} \mathrm{NH}_{4}{ }^{+} / \mathrm{C}$ oxidable/MO. Los resultados obtenidos mostraron coeficientes de correlación de Pearson bajos. Se concluye que las variables $\mathrm{C}$ oxidable y MO están
\end{abstract}

1 Producto derivado del proyecto de investigación "Análisis de la concentración de $\mathrm{C}, \mathrm{N}$, su relación $\mathrm{C} / \mathrm{N}$ en suelo, y su influencia sobre el contenido de nitratos en aguas superficiales del municipio de Zona Bananera (Magdalena)". Presentado por el Grupo de Investigación Suelo Ambiente y Sociedad, de la Universidad del Magdalena.

D. V. Escorcia, Universidad del Magdalena, Santa Marta, Colombia, email: dayanaescorciavo@unimagdalena.edu.co.

S. E. Aguirre, Universidad del Magdalena, Santa Marta, Colombia, email: saguirre@unimagdalena.edu.co.

J. J. Vargas, Universidad del Magdalena, Santa Marta, Colombia, email: jvargass@unimagdalena.edu.co.

Como citar este artículo: Escorcia, D. V., Aguirre, S. E. y Vargas, J. J. Incidencia de la profundidad de muestreo sobre el contenido de formas de carbono y nitrógeno en suelos de la Zona Bananera del departamento del Magdalena (Colombia), Entre Ciencia e Ingeniería, vol. 15, no. 30, pp.63-70, julio-diciembre 2021. DOI: https://doi.org/ 31908/19098367.2586.

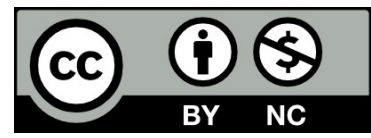

Attribution-NonCommercial 4.0 Intenational (CC By-NC 4.0) fuertemente relacionadas, mientras que las demás presentan una relación no lineal.

Palabras clave-Carbono, Nitrógeno, profundidad de muestreo, suelos, Zona Bananera.

Abstract-The objective of the work was to determine the incidence of the sampling depth on the carbon and nitrogen content in soils of the municipality of Zona Bananera of the department of Magdalena (Colombia). Data analysis was performed with statistical software Infostat v.2008 and $R$ v.3.5.2, using multivariate analysis of variance, Pearson's correlation analysis, and descriptive statistics of each variable. The results show significant differences $(p<0,05)$ between the depth groups for the selected variables, with lower contents found at depth 2 . In addition, there is a significant contribution of the variables to the statistical differences between the groups, except for ammonium and nitrate. Correlation is evident between the variables $\mathrm{C}$ oxidizable/MO, $\mathrm{NO}_{3}{ }^{-} / \mathrm{NO}_{2}{ }^{-}$, and $\mathrm{NH}_{4}{ }^{+} / \mathrm{C}$ oxidizable/MO. The results obtained showed low Pearson correlation coefficients. It is concluded that the variables $\mathrm{C}$ oxidizable and MO are firmly related, while the others present a non-linear relationship.

Keywords-Carbon, Nitrogen, sampling depth, soils, Zona Bananera.

\section{INTRODUCCIÓN}

Q L SUELO es considerado un sistema que enlaza factores bióticos y abióticos del ambiente, y contiene sustancias inorgánicas que son de vital importancia en el desarrollo agrícola. El agua, los nutrientes minerales, el dióxido de carbono $\left(\mathrm{CO}_{2}\right)$, oxígeno $\left(\mathrm{O}_{2}\right)$, entre otros, son elementos absorbidos por las plantas para el proceso de fotosíntesis. Los nutrientes presentes en el suelo suelen dividirse en macronutrientes y micronutrientes teniendo en cuenta las concentraciones en las que estos son requeridos; Carbono (C), 
Hidrógeno $(\mathrm{H})$, Oxígeno $(\mathrm{O})$, Nitrógeno $(\mathrm{N})$, Fósforo $(\mathrm{P})$, Azufre (S), Potasio (K), Calcio (Ca), Magnesio (Mg) y Silicio (Si) son requeridos en mayores concentraciones, mientras Hierro (Fe), Manganeso (Mn), Cobre (Cu), Zinc ( $\mathrm{Zn})$, Molibdeno (Mo), Boro (B), Cloro (Cl), Níquel (Ni) y Sodio (Na) son necesarios en menores proporciones. De hecho, el desarrollo de las raíces de los cultivos está muy relacionado con la disponibilidad y distribución de estos nutrientes en el perfil del suelo, por lo que se argumenta heterogeneidad [1], y es normal encontrar concentraciones diferentes en distancias mínimas [2]. La productividad y la dinámica de los ecosistemas terrestres están altamente relacionadas y algunas veces limitadas por la disponibilidad de nutrientes en el suelo [3].

El nitrógeno se encuentra en forma orgánica e inorgánica distribuidas heterogéneamente en el suelo, y se estima que más del $90 \%$ corresponde a nitrógeno orgánico producido por la degradación de material vegetal y restos de animales [4]. Por esta razón, una amplia variedad de microorganismos interviene sobre su dinámica en la biosfera [5] y abarcan procesos de fijación de nitrógeno atmosférico $N_{2}$, mineralización, nitrificación, desnitrificación [6] y oxidación anaeróbica del amonio [7]. Según [8], este nutriente es fundamental para el desarrollo y productividad de los cultivos y es absorbido como nitrato, nitrito y amonio a través de las raíces; siendo el nitrato la forma más móvil en el suelo y también la que se pierde con mayor facilidad por lixiviación [1].

La fertilidad de los suelos se logra cuando estos disponen de los contenidos adecuados de nutrientes esenciales [9]; no obstante, las cantidades que se encuentran naturalmente en ellos no logran suplir los requerimientos nutricionales y por esto son empleados fertilizantes naturales orgánicos y químicos, en especial nitrogenados [10], [11]. Estas fuentes nitrogenadas generan importantes cambios químicos sobre el recurso y dependen de la dinámica del elemento nutriente, la dosis y fuente utilizada, el sistema de aplicación, las características del suelo y las condiciones climáticas. Así mismo, el aumento en los niveles de determinado nutriente, su persistencia y residualidad en el tiempo pueden modificar las reacciones que tienen lugar en él y también la disponibilidad de otros elementos esenciales para la nutrición, influyendo sobre la productividad de las cosechas y afectando la calidad del medio ambiente [12].

Gruber y Galloway (2008) citado por [13] exponen que la acción humana genera cambios en el ciclo del nitrógeno que provocan su acumulación en la atmósfera, suelo y agua, y afectan procesos importantes en los ciclos de elementos como el fósforo y carbono. Según [14], esto genera graves problemas ambientales a nivel mundial y ocasiona impactos negativos como la escasez de agua, pérdida de biodiversidad, inundaciones, perdida de la fertilidad de los suelos, erosión, desequilibrio de nutrientes y pérdida del Carbono Orgánico del Suelo (COS).

En los procesos que se presentan dentro del ciclo del carbono existe un intercambio de sus diferentes formas entre la atmósfera, el océano, la biosfera terrestre y los depósitos geológicos. Es importante resaltar que la retención de carbono se produce cuando este es absorbido y almacenado en el suelo, hecho que contribuye significativamente al cambio climático debido a que cuanto mayor sea el contenido de carbono almacenado, menores serán las cantidades de dióxido de carbono en la atmósfera [15]. El COS ocupa casi un $70 \%$ del carbono orgánico presente en la biosfera [16]; esto indica en palabras de [17] que el suelo actúa como reservorio potencial de este nutriente dependiendo de su uso y manejo, y otros factores como el contenido/tipo de arcillas, la precipitación, temperatura, radiación solar, la densidad y profundidad del suelo [18]. Esta forma de carbono es desestabilizada por diferentes mecanismos a medida que aumenta la profundidad en el perfil, por esto resulta crucial conocer su distribución vertical en un área determinada [19]. De hecho, en la agricultura se producen pérdidas que varían entre el 30 y $50 \%$ debido a la incorporación de nuevos suelos con sistemas intensivos de cultivo, constituyendo una fuente de dióxido de carbono para la atmósfera que es producido por la mineralización de la Materia Orgánica del Suelo (MOS) y el metabolismo de las raíces de las plantas. Además, la presencia de este macronutriente en el suelo afecta sus propiedades fisicoquímicas y biológicas relacionadas con calidad, sustentabilidad y capacidad productiva [20].

Niveles bajos de carbono afectan la agricultura y la productividad como consecuencia de la baja fertilidad del suelo; por ello en la actualidad el hombre ha optado por mejorar su uso y manejo incorporando materiales orgánicos beneficiosos, a fin de lograr un equilibrio y satisfacer las demandas de este nutriente [15]. De la misma manera, [21] destaca el proceso de erosión del suelo y la deposición de macronutrientes, especialmente el COS, debido a que algunos de los sedimentos generados se redistribuyen sobre el paisaje y otros se depositan en terrenos con fuertes pendientes y también en los sistemas acuáticos. No obstante, el carbono que no es transportado por la erosión es emitido a la atmósfera en forma de dióxido de carbono o metano 4 , por procesos de mineralización y metanogénesis; respectivamente. El uso de herbicidas, plaguicidas y pesticidas para aumentar de manera indirecta la productividad de los cultivos afecta gravemente los ciclos de regulación del carbono y ocasiona aumento en las concentraciones de gases de efecto invernadero (GEI) en la atmósfera que genera contaminación a largo plazo y un significativo aporte al cambio climático a nivel mundial [22], [15].

Por otro lado, la MOS es un indicador clave de la calidad del recurso en la producción de cultivos y la captura de carbono, y es el principal determinante de su actividad biológica. Este último aspecto tiene gran influencia sobre sus propiedades fisicoquímicas, donde altos contenidos de materia orgánica aumentan la agregación y estabilidad de su estructura, mejoran la tasa de infiltración y disponibilidad de agua, así como la resistencia contra la erosión hídrica y eólica. La materia orgánica también mejora la dinámica y biodisponibilidad de los principales nutrientes de las plantas [23]. Es necesario tener en cuenta que la aplicación de nitrógeno y MOS debe dosificarse a lo largo de todo el cultivo 
teniendo en cuenta las condiciones climáticas, las etapas fenológicas de los cultivos y el comportamiento del material vegetal [24]. Teniendo en cuenta lo anterior, el objetivo de este trabajo fue determinar la incidencia que tiene la profundidad de muestreo sobre el contenido de las formas de carbono y nitrógeno en los suelos del municipio de Zona Bananera del departamento del Magdalena (Colombia).

\section{MetodologíA}

La metodología se presenta en tres subsecciones: en la primera se describe el área de estudio; en la segunda se definen los aspectos concernientes al muestreo y caracterización analítica de las variables definidas con sus respectivos métodos de análisis; y en la tercera se muestran los métodos y técnicas estadísticas seleccionadas para el análisis de los datos.

\section{A. Área de estudio}

La investigación fue realizada al noreste del departamento del Magdalena, en el municipio de Zona Bananera considerado a partir de estudios previos de [25] como una de las zonas con cultivos de mayor importancia en esta área, localizado entre las coordenadas $10^{\circ} 46^{\prime} 00^{\prime \prime}$ Latitud Norte y $74^{\circ} 08^{\prime} 00^{\prime \prime}$ Longitud Oeste a una altura de 20 m.s.n.m. Se encuentra en un área situada entre la Sierra Nevada de Santa Marta y la Ciénaga Grande, con temperatura promedio de $27^{\circ} \mathrm{C}$, humedad relativa del $82 \%$ y precipitación de 1371 $\mathrm{mm} / \mathrm{año}$. Se caracteriza por ser una zona eminentemente agrícola con cultivos de banano para exportación, palma de aceite, arroz, cacao y café, entre otros [26]. Además, presenta suelos de origen aluvial, profundos de más de $1,2 \mathrm{~m}$ con drenaje de pobre a bueno, texturas francas, franco arcillosas y arenosas, $\mathrm{pH}$ entre 6 y 7, buena fertilidad y contenidos variables de materia orgánica con tendencia a ser bajos en algunas áreas con problemas de salinidad [25].

\section{B. Muestreo y caracterización analítica}

Para el estudio fue realizado un muestreo aleatorio simple con recolección de muestras simples de suelo a diferentes profundidades, de $0-10 \mathrm{y}$ de $10-20 \mathrm{~cm}$, en áreas cultivadas con banano, palma africana, y árboles frutales. Cada punto muestreado dentro de la zona se identificó con sus respectivas coordenadas geográficas utilizando GPS y se determinaron los parámetros químicos presentados en la Tabla I por medio de análisis en el Laboratorio de Servicios A nalíticos del Centro Internacional de Agricultura Tropical (CIAT) en Palmira.

TABLA I

PARÁMETROS Y MÉTODOS EN SUELO

\begin{tabular}{|c|c|c|c|}
\hline Parámetro & Simbolo & Unidad & Método \\
\hline Carbono oxidable & C oxidable & $\mathrm{g} / \mathrm{kg}$ & \multirow{2}{*}{ Auto-analizadores } \\
\hline Materia orgánica & $\mathrm{MO}$ & $\mathrm{g} / \mathrm{kg}$ & \\
\hline Nitrógeno total & $\mathrm{N}$-total & $\mathrm{g} / \mathrm{kg}$ & \\
\hline Amonio & $\mathrm{NH}_{4}{ }^{+}$ & $\mathrm{mg} / \mathrm{kg}$ & \multirow{2}{*}{ Espectrofotometría UV-VIS (Equipo-Skalar) } \\
\hline Nitrito & $\mathrm{NO}_{2}^{-}$ & $\mathrm{mg} / \mathrm{kg}$ & \\
\hline Nitrato & $\mathrm{NO}_{3}^{-}$ & $\mathrm{mg} / \mathrm{kg}$ & \\
\hline
\end{tabular}

\section{Análisis de los datos}

Para las variables definidas en la investigación, relacionadas con las formas de carbono y nitrógeno, se realizó un Análisis de Varianza Multivariado (MANOVA) [27], [28] utilizando las profundidades en el perfil del suelo como grupos de comparación para establecer las posibles diferencias estadísticas entre ellos. Así mismo, se llevó a cabo un análisis de correlación de Pearson para determinar las posibles relaciones entre los elementos nutrientes y su nivel de asociación, así como también la estadística descriptiva de cada variable. Para el análisis de los datos fue utilizado el software estadístico InfoStat versión 2008 y R versión 3.5.2.

\section{RESULTADOS Y DISCUSIÓN}

Mediante el análisis de varianza multivariado se comprobó si existían diferencias significativas entre los grupos de profundidad para las variables/parámetros químicos seleccionados. Para ello, fueron utilizados estadísticos de prueba los cuales son: la traza de Pillai, la traza de LawleyHotelling, la lambda de Wilks y la raíz máxima de Roy. En la Tabla II se presentan los valores obtenidos para los estadísticos, cada uno de los cuales tiene la forma de transformarse a una distribución $\mathrm{F}$, observándose en este caso un valor $\mathrm{F}$ de 2,6937 en todas las pruebas. Los resultados encontrados fueron estadísticamente significativos considerando que el análisis mostró un $\mathrm{p}$ valor igual a 0,0351 menor al nivel de significación $\alpha=0,05$ rechazando la hipótesis nula de que no existen diferencias significativas entre los grupos.

TABLA II

ESTADÍSTICOS DE PRUEBA PARA MANOVA

\begin{tabular}{|c|c|c|c|c|c|c|}
\hline $\begin{array}{c}\text { Factor de } \\
\text { variación }\end{array}$ & Prueba & Estadistico & F & gl (num) & gl (den) & p \\
\hline \multirow{4}{*}{ Profundidad } & Lambda de Wilks & 0,625 & & & & \\
\cline { 2 - 4 } & Traza de Pillai & 0,374 & \multirow{2}{*}{2,6937} & 6 & 27 & 0,0351 \\
\cline { 2 - 7 } & $\begin{array}{c}\text { Traza de Lawley- } \\
\text { Hotelling }\end{array}$ & 0,598 & & & & \\
\cline { 2 - 6 } & Raíz máxima de Roy & 0,598 & & & & \\
\hline
\end{tabular}

Así mismo, la Tabla III muestra las diferencias significativas entre los grupos de profundidad a partir de la prueba Hotelling, usando un nivel de confianza del 95\%. En ella se presenta el mismo número de muestras $(\mathrm{n}=17)$ para cada profundidad de muestreo (con un total de 34 muestras) y la última columna indica que, al ser estas letras no comunes, las medias obtenidas resultan ser significativamente diferentes $(\mathrm{p}<0,05)$ entre los grupos. Además, en todos los casos se observa que los contenidos de nutrientes en la profundidad de 10-20 cm fueron menores en comparación con los obtenidos en los primeros $10 \mathrm{~cm}$ de suelo. 
TABLA III

DIFERENCIAS SIGNIFICATIVAS ENTRE LOS GRUPOS DE PROFUNDIDAD USANDO LA PRUEBA HOTELLING $(\mathrm{GL}=32 ; \alpha=0,05)$

\begin{tabular}{|c|c|c|c|c|c|c|c|c|}
\hline Profundidad & C oxidable & MO & N-total $^{-}$ & $\mathbf{N H}_{4}{ }^{+}$ & $\mathbf{N O}^{-}$ & $\mathbf{N O}^{-}$ & $\mathbf{n}$ & \\
\hline 1 & 15,90 & 36,10 & 1,16 & 8,47 & 38,78 & 18,00 & 17 & $\mathrm{~A}$ \\
\hline 2 & 11,22 & 25,52 & 0,73 & 5,39 & 24,21 & 10,36 & 17 & $\mathrm{~B}$ \\
\hline
\end{tabular}

Teniendo en cuenta que los resultados obtenidos evidenciaron que existen diferencias entre ambos grupos, se procedió a analizar si las diferencias producidas entre vectores son explicadas por las diferencias que se producen entre las medias de alguna de esas variables. Para este caso, se realizó un análisis univariante de cada uno de los parámetros químicos (formas de carbono y nitrógeno) para conocer en donde se presentan las diferencias significativas. Esto permitió obtener la contribución de cada variable a las diferencias entre grupos, presentadas en la Tabla IV. En ella los datos indican que las variables del estudio tienen una contribución significativa a las diferencias estadísticas entre los grupos, debido a que los $\mathrm{p}$ valores fueron menores al nivel de significación de 0,05 exceptuando los casos específicos para el amonio y el nitrato en los que se encontraron valores de $\mathrm{p}$ igual a 0,1783 y 0,0605 ; respectivamente. En otras palabras, se puede concluir que las diferencias entre los vectores de medias son debidas a las diferencias entre medias de alguna de las variables.

TABLA IV

CONTRIBUCIÓN DE CADA VARIABLE A LAS DIFERENCIAS ENTRE GRUPOS

\begin{tabular}{|c|c|c|}
\hline Variable & $\mathbf{F}$ & $\mathbf{p}$ \\
\hline C oxidable & 6,635 & 0,0148 \\
\hline $\mathrm{MO}$ & 6,582 & 0,0151 \\
\hline $\mathrm{N}$-total & 6,389 & 0,0166 \\
\hline $\mathrm{NH}_{4}{ }^{-}$ & 1,894 & 0,1783 \\
\hline $\mathrm{NO}_{3}^{-}$ & 3,784 & 0,0605 \\
\hline $\mathrm{NO}_{2}^{-}$ & 5,664 & 0,0234 \\
\hline
\end{tabular}

Por otro lado, al aplicar el análisis de correlación de Pearson a las variables del estudio se obtuvieron los coeficientes de correlación presentados en la Tabla $\mathrm{V}$ y Tabla VI para las profundidades 1 y 2; respectivamente. En ambos casos se encontró que las variables $\mathrm{C}$ oxidable y $\mathrm{MO}$ tienen una relación lineal perfecta $(r=1,00)$ lo que indica que ambas variables tienden a aumentar o disminuir en la misma proporción. De la misma manera, las variables $\mathrm{NO}_{3}{ }^{-}$y $\mathrm{NO}_{2}{ }^{-}$ presentaron una relación positiva grande con coeficientes superiores a 0,80 y $\mathrm{p}$ valores menores al nivel de significación $\alpha=0,05$ lo que evidencia que las correlaciones fueron estadísticamente significativas. Para el caso del amonio $\left(\mathrm{NH}_{4}{ }^{+}\right)$en la profundidad 2 se obtuvo una relación positiva con las variables $\mathrm{C}$ oxidable y $\mathrm{MO}$, presentándose un coeficiente de correlación $\mathrm{r}=0,545$ que explica una asociación de moderada a fuerte entre estas formas de nutrientes. No obstante, para aquellos resultados donde se obtuvieron coeficientes de correlación de Pearson bajos, es importante resaltar que esto no representa la no asociación entre las variables, por el contrario, se denota que pueden estar fuertemente relacionadas entre sí, pero de forma indirecta no lineal.
TABLA V

CORRELACIONES DE PEARSON PARA FORMAS DE CARBONO Y NITRÓGENO PARA LA PROFUNDIDAD 1

\begin{tabular}{|c|c|c|c|c|c|c|}
\hline & C oxidable & MO & N-total & $\mathbf{N H}_{4}{ }^{+}$ & $\mathbf{N O}^{-}{ }^{-}$ & $\mathbf{N O}_{2}{ }^{-}$ \\
\hline C oxidable & 1,00 & 0,00 & 0,184 & 0,259 & 0,296 & 0,861 \\
\hline MO & $1,00^{*}$ & 1,00 & 0,186 & 0,259 & 0,296 & 0,862 \\
\hline N-total & 0,338 & 0,337 & 1,00 & 0,753 & 0,804 & 0,687 \\
\hline NH $_{4}{ }^{+}$ & 0,290 & 0,290 & 0,082 & 1,00 & 0,958 & 0,532 \\
\hline $\mathbf{N O}_{3}{ }^{-}$ & $-0,269$ & $-0,269$ & $-0,065$ & $-0,014$ & 1,00 & 0,000027 \\
\hline $\mathbf{N O}_{2}{ }^{-}$ & 0,046 & 0,046 & $-0,106$ & 0,163 & $0,838^{*}$ & 1,00 \\
\hline
\end{tabular}

* La correlación es significativa al nivel 0,05

TABLA VI

CORRELACIONES DE PEARSON PARA FORMAS DE CARBONO Y NITRÓGENO PARA LA PROFUNDIDAD 2

\begin{tabular}{|c|c|c|c|c|c|c|}
\hline & C oxidable & MO & N-total & $\mathbf{N H}_{4}{ }^{+}$ & $\mathbf{N O}_{3}{ }^{-}$ & $\mathbf{N O}^{-}$ \\
\hline C oxidable & 1,00 & 0,00 & 0,924 & 0,024 & 0,540 & 0,447 \\
\hline MO & $1,00^{*}$ & 1,00 & 0,923 & 0,024 & 0,542 & 0,452 \\
\hline N-total & 0,025 & 0,025 & 1,00 & 0,182 & 0,337 & 0,471 \\
\hline $\mathbf{N H}_{4}{ }^{+}$ & $0,545^{*}$ & $0,545^{*}$ & $-0,339$ & 1,00 & 0,963 & 0,798 \\
\hline $\mathbf{N O}_{3}{ }^{-}$ & 0,160 & 0,159 & 0,248 & $-0,012$ & 1,00 & 0,0000002 \\
\hline $\mathbf{N O}_{2}{ }^{-}$ & 0,198 & 0,196 & 0,188 & 0,067 & $0,918^{*}$ & 1,00 \\
\hline
\end{tabular}

* La correlación es significativa al nivel 0,05

La Fig. 1 y Fig. 2 presentan los diagramas de caja y bigotes correspondientes a las variables carbono oxidable y materia orgánica para ambas profundidades de muestreo. En ellas se observa el mismo comportamiento para ambos parámetros, lo cual explica la relación lineal perfecta obtenida en la prueba de correlación. También se observa que los contenidos medios de los grupos son estadísticamente diferentes y que para la profundidad 1 las concentraciones de $\mathrm{C}$ oxidable y MO tienen mayor dispersión (variabilidad) que las encontradas en la profundidad 2.

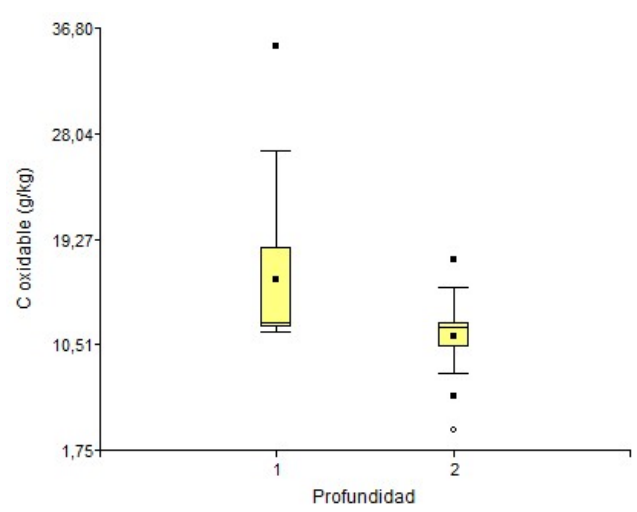

Fig. 1. Carbono oxidable v/s Profundidad

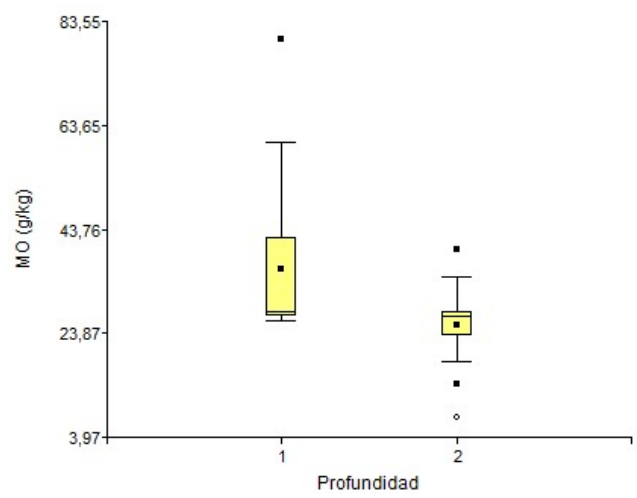

Fig. 2. Materia orgánica v/s Profundidad 
En la Fig. 3 y Fig. 4 se observa el comportamiento de las variables nitrógeno total y amonio en cada profundidad muestreada. Para la Fig. 3, el diagrama de caja y bigotes muestra diferencias entre los contenidos medios de los grupos para esta variable y también entre las dispersiones de cada uno de ellos. Por otra parte, en la Fig. 4 se presentó poca variación de los datos correspondientes al amonio e igualdad entre los contenidos medios de nutriente de las profundidades 1 y 2 , lo cual es coherente con el $p$ valor obtenido superior a 0,05 que indica que no existen diferencias significativas entre los grupos para este parámetro.

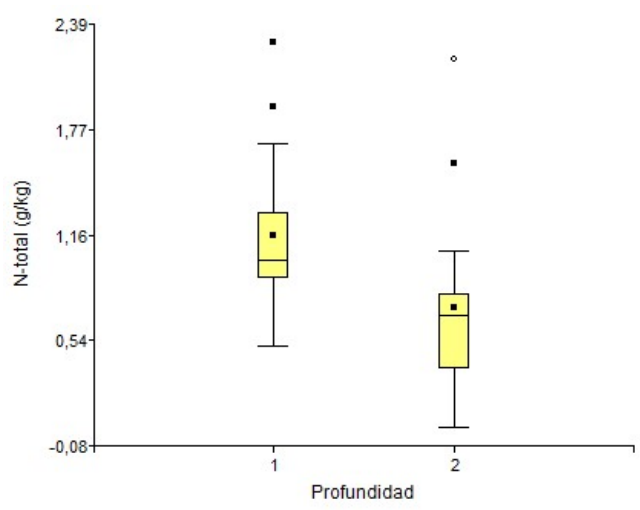

Fig. 3. Nitrógeno total v/s Profundidad

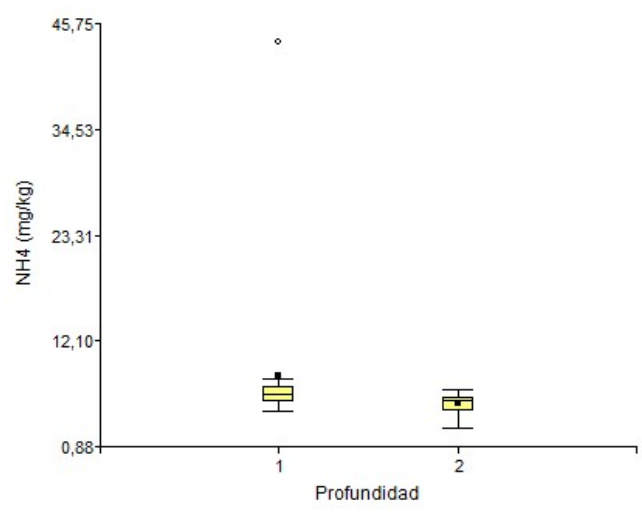

Fig. 4. Amonio v/s Profundidad

Finalmente, en la Fig. 5 y Fig. 6 se presentan los diagramas de caja y bigotes correspondientes a las variables nitrato y nitrito para ambos grupos de profundidad, encontrándose en la Fig. 5 similitudes en el comportamiento (dispersión) de los datos y presentando diferencias entre los contenidos medios de los grupos. La variable nitrito es explicada por el contenido de nitrato, debido a que el comportamiento entre ellas también resultó ser similar con la diferencia de que para la profundidad 1 en la Fig. 6 se presentaron grandes variaciones en los datos que pueden ser explicadas por datos atípicos presentes en el conjunto de valores obtenidos a partir del muestreo en campo, que a su vez pudo estar influenciado por otras variables externas que no podían ser controladas.

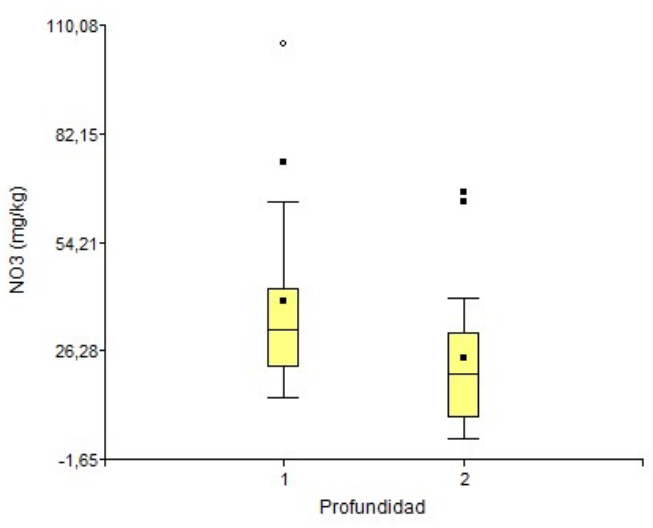

Fig. 5. Nitrato v/s Profundidad

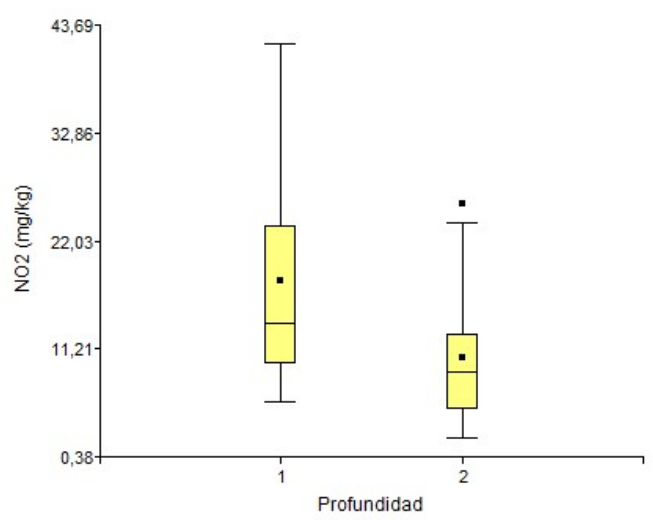

Fig. 6. Nitrito v/s Profundidad

Por otro lado, el análisis de varianza multivariado debe cumplir el supuesto básico de normalidad en las $k$ poblaciones, razón por la cual fue aplicado al conjunto de datos la prueba de Mardia para cada grupo de profundidad del suelo; esto hizo posible identificar si la asimetría y la curtosis multivariantes del conjunto de variables del estudio permiten asumir o no la hipótesis de normalidad.

En este sentido, resultó importante verificar la normalidad univariante como condición necesaria pero no suficiente para que los datos de manera conjunta sigan una distribución normal multivariante. Lo anterior indica que, si dicha distribución conjunta es normal multivariada, cada una de las marginales es una normal univariante, aclarando que este comportamiento no puede aceptarse a la inversa [29]. Para este estudio se comprobó en primer lugar que todas las variables individuales se distribuyen normalmente mediante la prueba de Shapiro-Wilks en la que se obtuvieron los resultados presentados en la Tabla VII para los datos originales por grupo de profundidad; los valores encontrados en la significación de los estadísticos permiten comprobar que no hay normalidad de las variables a excepción del $\mathrm{N}$-total y el $\mathrm{NH}_{4}{ }^{+}$donde el $\mathrm{p}$ valor fue mayor al nivel de significancia $\alpha=0,05$, aceptando la hipótesis nula de normalidad de los datos para los grupos 1 y 2; respectivamente. En concordancia con lo anterior, se encontró que al aplicar la prueba de Mardia al grupo 1 las variables no cumplen con el supuesto de normalidad multivariada, en contraste con el grupo 2 que se comporta como una distribución normal, tal como se observa en la Tabla VIII. 
TABLA VII

PRUEBA DE NORMALIDAD DE SHAPIRO-WILKS APLICADA A DATOS ORIGINALES POR GRUPO DE PROFUNDIDAD

\begin{tabular}{|c|c|c|c|c|}
\hline \multirow{2}{*}{ Variable } & \multicolumn{4}{|c|}{ Datos originales } \\
\cline { 2 - 5 } & \multicolumn{2}{|c|}{ Grupo l } & \multicolumn{2}{c|}{ Grupo 2 } \\
\cline { 2 - 5 } & Estadístico & p valor & Estadistico & p valor \\
\hline C oxidable & 0,6882 & 0,0001 & 0,8880 & 0,0430 \\
\hline $\mathrm{MO}$ & 0,6878 & 0,0001 & 0,8864 & 0,0404 \\
\hline $\mathrm{N}-$ total & 0,9043 & 0,0802 & 0,8679 & 0,0203 \\
\hline $\mathrm{NH}_{4}{ }^{-}$ & 0,3623 & $<0,001$ & 0,9528 & 0,5028 \\
\hline $\mathrm{NO}_{3}^{-}$ & 0,8537 & 0,0122 & 0,8674 & 0,0200 \\
\hline $\mathrm{NO}_{2}^{-}$ & 0,8791 & 0,0308 & 0,8859 & 0,0396 \\
\hline
\end{tabular}

TABLA VIII

MEDIDAS ESTADÍSTICAS UNIVARIANTES DE LOS DATOS ORIGINALES POR GRUPO DE PROFUNDIDAD

\begin{tabular}{|c|c|c|c|c|}
\hline \multirow{2}{*}{ Variable } & \multicolumn{4}{|c|}{ Datos originales } \\
\cline { 2 - 5 } & \multicolumn{2}{|c|}{ Grupo l } & \multicolumn{2}{c|}{ Grupo 2 } \\
\cline { 2 - 5 } & Simetría & curtosis & Simetría & Curtosis \\
\hline C oxidable & 1,5663 & 1,4246 & $-0,5714$ & 0,6164 \\
\hline $\mathrm{MO}$ & 1,5664 & 1,4236 & $-0,5820$ & 0,6107 \\
\hline $\mathrm{N}-$ total $^{-}$ & 0,9400 & 0,0043 & 1,3152 & 1,4102 \\
\hline $\mathrm{NH}_{4}{ }^{-}$ & 3,3525 & 10,0185 & $-0,4874$ & $-0,7423$ \\
\hline $\mathrm{NO}_{3}^{-}$ & 1,2281 & 0,7533 & 1,0393 & 0,0813 \\
\hline $\mathrm{NO}_{2}^{-}$ & 0,8673 & $-0,4404$ & 0,8104 & $-0,5921$ \\
\hline
\end{tabular}

Teniendo en cuenta que el cumplimiento de este supuesto es necesario para garantizar la validez de los resultados, se aplicó la transformación Box Cox al conjunto de datos utilizando el software estadístico Minitab versión 2019 para cada variable. Se realizaron nuevamente las pruebas de normalidad y homocedasticidad para verificación de supuestos, y el análisis de varianza multivariado con los datos transformados. La Tabla IX, evidencia que la prueba de Shapiro-Wilks aplicada a los datos arrojó p-valores mayores al nivel de significancia $\alpha=0,05$ únicamente para las variables $\mathrm{N}$-total, $\mathrm{NO}_{3}{ }^{-}$y $\mathrm{NO}_{2}{ }^{-}$en ambos grupos de profundidad; esto indica la aceptación de la hipótesis nula de normalidad en comparación con los parámetros $\mathrm{C}$ oxidable, $\mathrm{MO} \mathrm{y} \mathrm{NH}_{4}^{+}$que no presentaron normalidad univariante. La prueba de normalidad multivariada de Mardia se cumplió para los dos grupos tal como se presenta en la Tabla X.

TABLA IX

PRUEBA DE NORMALIDAD DE SHAPIRO-WILKS APLICADA A DATOS TRANSFORMADOS POR GRUPO DE PROFUNDIDAD

\begin{tabular}{|c|c|c|c|c|}
\hline \multirow{2}{*}{ Variable } & \multicolumn{4}{|c|}{ Datos transformados } \\
\cline { 2 - 5 } & \multicolumn{2}{|c|}{ Grupo l } & \multicolumn{2}{c|}{ Grupo 2 } \\
\cline { 2 - 5 } & Estadístico & p valor & Estadístico & p valor \\
\hline C oxidable & 0,7151 & 0,0002 & 0,8402 & 0,0076 \\
\hline $\mathrm{MO}$ & 0,7147 & 0,0002 & 0,8385 & 0,0072 \\
\hline $\mathrm{N}_{\text {-total }}$ & 0,9486 & 0,4344 & 0,9647 & 0,7210 \\
\hline $\mathrm{NH}_{4}^{+}$ & 0,8584 & 0,0144 & 0,8359 & 0,0066 \\
\hline $\mathrm{NO}_{3}^{-}$ & 0,9636 & 0,7006 & 0,9704 & 0,8246 \\
\hline $\mathrm{NO}_{2}^{-}$ & 0,9621 & 0,6717 & 0,9529 & 0,5033 \\
\hline
\end{tabular}

TABLA X

MEDIDAS ESTADÍSTICAS UNIVARIANTES DE LOS DATOS TRANSFORMADOS POR GRUPO DE PROFUNDIDAD

\begin{tabular}{|c|c|c|c|c|}
\hline \multirow{3}{*}{ Variable } & \multicolumn{4}{|c|}{ Datos transformados } \\
\cline { 2 - 5 } & \multicolumn{2}{|c|}{ Grupo l } & \multicolumn{2}{c|}{ Grupo 2 } \\
\cline { 2 - 5 } & Simetría & curtosis & Simetría & Curtosis \\
\hline C oxidable & 1,3634 & 0,6217 & $-1,1542$ & 1,4375 \\
\hline $\mathrm{MO}$ & 1,3638 & 0,6217 & $-1,1603$ & 1,4341 \\
\hline $\mathrm{N}-$ total $^{+}$ & 0,5931 & $-0,4145$ & 0,2638 & 0,2219 \\
\hline $\mathrm{NH}_{4}{ }^{-}$ & $-1,3935$ & 2,5563 & 1,4505 & 1,8307 \\
\hline $\mathrm{NO}_{3}^{-}$ & 0,2672 & $-0,9617$ & $-0,2447$ & $-0,9872$ \\
\hline $\mathrm{NO}_{2}^{-}$ & 0,1419 & $-1,2619$ & $-0,1419$ & $-1,2414$ \\
\hline
\end{tabular}

Así mismo, fue comprobado el supuesto de homocedasticidad mediante la prueba $\mathrm{M}$ de Box, cuyo resultado representado en una variable aleatoria $\mathrm{U}$ se comparó con una chi cuadrado de $v=21$ grados de libertad. Al aplicar la prueba en el software estadístico R se obtuvo un valor de $\mathrm{U}$ igual a 64,049 mayor al valor crítico 32,67 correspondiente a un $\alpha=0,05$ de dicha distribución; razón por la cual se rechazó la hipótesis nula de igualdad de varianzas. De la misma manera, es válido indicar que fueron utilizados los datos transformados para cumplir el supuesto de homogeneidad de varianzas encontrándose un valor de $\mathrm{U}=27,662$ menor al valor critico en el nivel de significancia definido para el análisis; esto permitió corroborar que los datos obtenidos a partir de la transformación Box Cox también cumplen con el supuesto de homocedasticidad.

Con el análisis de varianza multivariado realizado a partir de los datos transformados se observó el mismo comportamiento encontrado en los datos originales. Esto permitió constatar los siguientes aspectos: 1) los resultados obtenidos a partir de los estadísticos de prueba son estadísticamente significativos considerando que el análisis mostró un $\mathrm{p}$ valor igual a 0,0136 menor al nivel de significancia $\alpha=0,05$, por lo que fue rechazada la hipótesis nula de que no existen diferencias significativas entre los grupos de profundidad y 2) en la prueba de Hotelling al nivel de confianza del $95 \%$ se observó que las medias obtenidas entre grupos son significativamente diferentes, obteniéndose mayores contenidos de las formas de carbono y nitrógeno en la profundidad de 0-10 $\mathrm{cm}$. No obstante, las diferencias producidas entre vectores de medias son explicadas por las diferencias que se producen entre las medias de las variables, siendo los $\mathrm{p}$ valores obtenidos menores al nivel de significación de 0,05 , lo cual indicó que todas las variables del estudio tienen una contribución significativa a las diferencias estadísticas entre los grupos de profundidades. Vale la pena considerar que los suelos hacen parte de un sistema holístico complejo (medio ambiente) donde hay diferentes interacciones entre la parte biótica y abiótica sometidos a fluctuaciones constantes, de las que se pueden mencionar temperatura, régimen de humedad, flujo constante de gases, pedogénesis, intervención antrópica, entre otras; condiciones que afectan el recurso edáfico por lo que presentan una amplia variabilidad estadística. 


\section{CONCLUSIONES}

El análisis de varianza multivariado permitió comprobar que existen diferencias significativas entre los grupos de profundidad para los parámetros químicos seleccionados. Además, al aplicar la prueba de Hotelling con relación a cada variable, usando un nivel de significación de 0,05 , se obtuvo que las medias obtenidas resultan ser significativamente diferentes $(\mathrm{p}<0,05)$ entre los grupos, siendo los contenidos de nutrientes en la profundidad de $10-20 \mathrm{~cm}$ menores a los obtenidos en los primeros $10 \mathrm{~cm}$ del suelo. Así mismo, el análisis univariante de cada una de las variables (formas de carbono y nitrógeno) evidenció que estas tienen una contribución significativa a las diferencias estadísticas entre los grupos, exceptuando los casos específicos para el amonio y el nitrato. Finalmente, al aplicar el análisis de correlación de Pearson se encontró que las variables $\mathrm{C}$ oxidable y $\mathrm{MO}$ tienen una relación lineal perfecta $(\mathrm{r}=1,00)$, las variables $\mathrm{NO}_{3}{ }^{-}$y $\mathrm{NO}_{2}{ }^{-}$ presentaron una relación positiva grande con coeficientes superiores a 0,80 y para el caso del amonio $\left(\mathrm{NH}_{4}^{+}\right)$en la profundidad 2 se obtuvo una relación positiva con las variables $\mathrm{C}$ oxidable y $\mathrm{MO}$ que explica la asociación entre estas formas de nutrientes. No obstante, los resultados donde se obtuvieron coeficientes de correlación de Pearson bajos probablemente indican que puede existir asociación entre las variables y estar fuertemente relacionadas entre sí, pero de indirecta no lineal.

\section{REFERENCIAS}

[1] T. Celis, "Efecto de la distribución heterogénea de nitrato sobre el sistema radical de Capsicum chinense Jacq", Tesis doctoral dirigida por I. Echevarría y M. Martínez, Centro de Investigación Científica de Yucatán, A.C., 27, sep. 2011.

[2] A. J. Bloom, J. Frensch and A. R. Taylor, "Influence of inorganic nitrogen and $\mathrm{pH}$ on the elongation of maize seminal roots", Ann BotLondon, 97, 867-873, 2006.

[3] L. Cerón y F. Aristizábal. (2012, Jul.). Dinámica del ciclo del nitrógeno y fósforo en suelos. Rev. Colomb. Biotecnol. [Online]. 14(1), pp. 285295. Disponible en: http://www.scielo.org.co/pdf/biote/v14n1/v14n1a26.pdf

[4] R. D. Zapata y W. Osorio, "La materia orgánica del suelo", en Ciencia del suelo: principios básicos, H. Burbano y F. Silva, Ed. Bogotá, Sociedad Colombiana de la Ciencia del Suelo (SCCS), 2010, pp. 357396.

[5] M. G. Klotz and L. Y. Stain, "Nitrifier genomics and evolution of the nitrogen cycle", FEMS Microbiological Letters, 278, 146-156, 2008.

[6] M. Hayatsu, K. Tago and M. Saito, "Various players in the nitrogen cycle: Diversity and functions of the microorganisms involved in nitrification and denitrification", Soil Science and Plant Nutrition, 54, $33-45,2008$.

[7] B. Hu, L. Shen, X. Xu and P. Zheng. (2011, Dec.). Anaerobic ammonium oxidation (anammox) in different natural ecosystems. Biochemical Society transactions. [Online]. 39(6), pp. 1811-6. Disponible en:

https://www.researchgate.net/publication/51818617 Anaerobic ammoni um_oxidation_anammox_in_different_natural_ecosystems

[8] M. Perea, Biotecnología: bananos y plátanos, Editora Guadalupe, Bogotá, 2003.

[9] M. E. Cruz, "Respuestas en desarrollo y producción del banano a la fertilización con nitrógeno y potasio en la zona de Santa Marta", Universidad Nacional de Colombia, Facultad de Ciencias Agrarias, 2014.

[10] J. Sánchez y J. Mira, Principios para la nutrición del cultivo de banano, AUGURA-CENIBANANO, Medellín, 2013, pp. 236.
[11] J. Torres, "Absorción, distribución y acumulación de nitrógeno en banano variedad Williams en dos ciclos de producción en zona húmeda tropical", Tesis doctoral dirigida por S. Magnitskiy, Facultad de Ciencias Agrarias, Universidad Nacional de Colombia, 2016.

[12] S. Sadeghian. (2003). Efecto de la fertilización con nitrógeno, fosforo, potasio y magnesio sobre las propiedades químicas de suelos cultivados en café. Cenicafé. [Online]. 54(3), pp. 242-257. Disponible en: https://www.cenicafe.org/es/publications/arc054\%2803\%29242-257.pdf

[13] A. R. Eugercios, M. Álvarez y E. Montero. (2017). Impactos de nitrógeno agrícola en los ecosistemas acuáticos. Ecosistemas. [Online]. 26(1), pp. 37-44. Disponible en:

https://www.revistaecosistemas.net/index.php/ecosistemas/article/view/1 309

[14] Food and Agriculture Organization (FAO), "Estado Mundial del Recurso Suelo", 2016. [Online]. Disponible: http://www.fao.org/3/ai5126s.pdf

[15] Food and Agriculture Organization (FAO), "Los suelos ayudan a combatir y adaptarse al cambio climático", 2015. [Online]. Disponible: http://www.fao.org/3/a-bb018s.pdf

[16] Food and Agriculture Organization (FAO), "Soil carbon sequestration for impoved land management", 2001. [Online]. Disponible: http://www.fao.org/3/a-bl001e.pdf

[17] R. Lal, "Residue management, conservation tillage and soil restoration for mitigating greenhouse effect by $\mathrm{CO}_{2}-$ enrichment", Soil Till. Res, 43, 81-107, 1997

[18] P. Etcheverría y D. Barahona, "Una base más estable: el secuestro de carbono en los suelos como alternativa de adaptación al cambio climático", 2017. [Online]. Disponible: https://www.inia.cl/wpcontent/uploads/2017/06/INIA-secuestro-de-carbono-91MundoAgro_Jun2017.pdf

[19] F. Paz y J. Etchevers, "Distribución a profundidad del carbono orgánico en los suelos de México", Terra Latinoamericana, 34, 339-355, 2016.

[20] E. Martínez, J. Fuentes y E. Acevedo, "Carbono orgánico y propiedades del suelo", R.C. Suelo Nutr. Veg., vol. 8, n. ${ }^{\circ}$ 1, pp. 68-96, 2008.

[21] R. Lal, "Soil Carbon Sequestration Impacts on Global. Climate Change and Food Security", Science AAAS, 304, 1623-1627, 2004.

[22] M. T. Colqué y V. E. Sánchez, "Los gases de efecto invernadero: ¿Por qué se produce el Calentamiento Global?", Asociación Civil Labor/Amigos de la Tierra-Perú, Mar. 2007. [Online]. Disponible: http://www.labor.org.pe/descargas/1 ra\%20publicacion_\%20abc\%20cc.p df

[23] Food and Agriculture Organization (FAO), "Captura de carbono en los suelos para un mejor manejo de la tierra", 2002. [Online]. Disponible: http://www.fao.org/3/a-bl001s.pdf

[24] J. Torres. (2017) Optimizan uso de nitrógeno en producción bananera. Agencia de noticias Universidad Nacional de Colombia. [Online]. Disponible:

https://agenciadenoticias.unal.edu.co/detalle/article/optimizan-uso-denitrogeno-en-produccion-bananera.html

[25] Instituto Geográfico Agustín Codazzi (IGAC), "Estudio general de suelos y zonificación de tierras. Departamento del Magdalena - Escala 1: 100000", Imprenta Nacional de Colombia, Bogotá D.C, 2009.

[26] O. Cabeza, "Agua y conflictos en la Zona Bananera del Caribe colombiano en la primera mitad del siglo XX", Tesis de maestría dirigida por S. Gallini, Facultad de Ciencias Económicas, Instituto de Estudios Ambientales, Universidad Nacional de Colombia, 2014.

[27] L. Díaz y M. Morales, Análisis Estadístico de datos Multivariados, Editorial de la Universidad Nacional de Colombia, Bogotá, 2012.

[28] K. V. Mardia, J. T. Kent and J. M. Bibby, Multivariate Analysis, Academic Press, London, 1979, pp. 518.

[29] N. González, J. Abad y J. P. Lévy, "Normalidad y otros supuestos en análisis de covarianzas", en Modelización con estructuras de covarianzas, J. Lévy, Ed. La Coruña, Netbiblo, 2006, pp. 31-57.

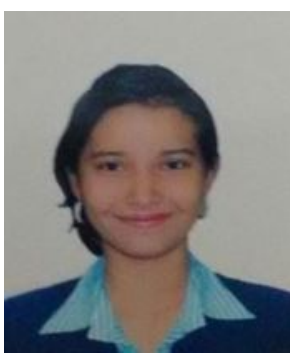

Dayana Vanessa Escorcia Ospino. Nació en Santa Marta, Colombia, el 28 de noviembre de 1996. Se graduó en la Universidad del Magdalena como Ingeniero Ambiental y Sanitario en 2018 y Magíster en Ingeniería en 2021.

En 2016 realizó un intercambio académico con la Escola Superior de Agricultura Luiz de Queiroz - ESALQ en São Paulo, Brasil. Además, desarrolló prácticas profesionales en el programa de Calidad Ambiental Marina del Instituto de 
Investigaciones Marinas y Costeras - INVEMAR en el año 2018, y en marzo de 2020 participó como conferencista en el XXXV Congreso Nacional de Ingeniería llevado a cabo en la ciudad de Cartagena, Colombia. ORCID: https://orcid.org/0000-0003-0895-6296.

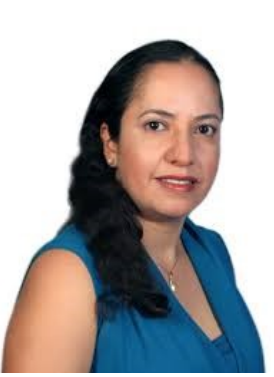

Sonia Esperanza Aguirre Forero. Nació en Sogamoso, Colombia, el 29 de enero de 1972. Se graduó en la Universidad Pedagógica y Tecnológica De Colombia - UPTC sede Tunja como Ingeniero Agrónomo en 1996 y especialista en 2002, en la Universidad Nacional de Colombia, sede Palmira, Valle como Magíster en Manejo y Conservación de Suelos en 2006 y como Doctor en Ciencias Agraria en 2014.

Ejerció profesionalmente en la Universidad Nacional Abierta y A Distancia, Universidad Pedagógica y Tecnológica de Colombia. Trabajó como consultora en proyectos de cambio climático de la Gobernación del Magdalena, CUC y cámara de comercio de Sucre, y para CORPOBOYACÁ en proyectos de conservación de suelos y siembra directa PROCAS. Actualmente es docente de planta de la Universidad del Magdalena. ORCID: https://orcid.org/0000-0002-6975-1940.

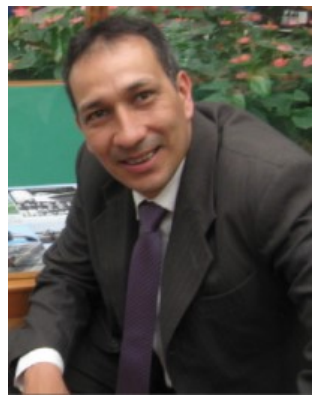

Jhon Jairo Vargas Sánchez. Nació en Manizales (Caldas), el 16 de junio de 1971. Doctor en Ingeniería de la Universidad Nacional de Colombia y Magíster en Investigación Operativa y Estadística de la Universidad Tecnológica de Pereira e Ingeniero Industrial de la Universidad Nacional de Colombia.

Docente de planta de la Facultad de Ingeniería de la Universidad del Magdalena. Director del grupo de Investigación en Estadística y Métodos cuantitativos de esta misma Universidad. Entre sus investigaciones se encuentran modelos de análisis envolvente de datos, estadística multivariada, series de tiempo y estadística aplicada. ORCID: https://orcid.org/0000-0003$3799-9850$. 